\title{
Fibers and fabrics for chemical and biological sensing
}

\author{
Shirley Coyle, Fernando Benito-Lopez, Tanja Radu, King-Tong Lau and Dermot Diamond* \\ National Centre for Sensor Research, CLARITY: Centre for Sensor Web Technologies, Dublin City \\ University, Glasnevin, Dublin 9, Ireland
}

\begin{abstract}
Wearable sensors can be used to monitor many interesting parameters about the wearer's physiology and environment, with important applications in personal health and well-being, sports performance and personal safety. Wearable chemical sensors can monitor the status of the wearer by accessing body fluids, such as sweat, in an unobtrusive manner. They can also be used to protect the wearer from hazards in the environment by sampling potentially harmful gas emissions such as carbon monoxide. Integrating chemical sensors into textile structures is a challenging and complex task. Issues which must be considered include sample collection, calibration, waste handling, fouling and reliability. Sensors must also be durable and comfortable to wear. Here we present examples of wearable chemical sensors that monitor the person and also their environment. We also discuss the issues involved in developing wearable chemical sensors and strategies for sensor design and textile integration.
\end{abstract}

Keywords: smart textiles, interactive materials, micro-fluidics, wearable chemical sensors

\section{Introduction}

Wearable sensors have the ability to monitor the physiology of the wearer and the surrounding environment. A wearable sensor may respond to physical changes such as body temperature or movements of the joints. A chemical sensor (chemosensor) detects and measures a particular chemical in its environment. Biosensors are closely related to chemical sensors, distinguished by the sensing element, which is biological in nature (e.g. enzyme, receptor, DNA, antibody, or microorganism). The integration of chemical sensors into a textile substrate is a challenging task as chemical sensors typically involve a molecular interaction or reaction in order to generate a signal. This involves delivery of a sample to an active sensor surface where the signal generation process happens. Often the sample is mixed with a reactive reagent, and mixing of samples and reagents results in waste products. Therefore a wearable chemosensor must be capable of controlling the movement of samples, reagents and (in some cases) waste in order to function.

The first section of this paper presents the development of wearable chemosensors to monitor sweat during exercise, "looking in" at the physiology of the wearer. The following section presents sensors which help to protect the wearer by "looking out" and detecting the presence of harmful gases (carbon monoxide and carbon dioxide in this case) which is of critical importance for those working in hazardous environments such as firefighters and first responders. The final section discusses the issues involved in integrating sensors with textiles and the requirements that must be considered when developing wearable chemosensors.

\section{Wearable chemical sensors "looking in" to monitor heath}

Wearable sensors ideally should offer a means to monitor the physiology of the wearer, in a continuous yet innocuous manner. The resulting information may be used to manage the wearer's health status through, for example, the detection of disease markers and to observe the outcome of prescribed treatment. It may also be used to improve fitness and optimize performance in sports and exercise, for example through monitoring of electrolyte loss through sweat.

For physiological assessment, blood is the best diagnostic medium, as it forms a delivery network for sustaining all cells of the body and it has been extensively studies for many years. Point of care diagnostics are performed with blood samples for assessing many health 
conditions, e.g. blood is tested for cholesterol levels to monitor cardiovascular health, glucose levels need to be regularly monitored to manage diabetes and blood lactate testing is one of the most commonly used methods for assessing fitness. Although blood carries many markers of interest it is impractical for wearable continuous monitoring due to the invasive nature of sampling. Therefore other body fluids, which can be accessed more easily through non- or minimally-invasive means, must be considered for analysis by wearable chemo-sensors. Urine, saliva, sweat, interstitial fluid, breath and tears are possible sampling sources (Brady et al., 2006).

Urine testing is a well established method typically implemented as a point of care solution wherein a dipstick containing a reagent changes colour depending on the concentration of a target species (Peredy and Powers, 1997). More recently, saliva has been identified as a "mirror of the body" for diagnostic testing (Lee et al., 2009, Kaufman E, 2002). However, for a wearable device to function autonomously, urine and saliva are difficult for continuous sample access. Tears have been used as an sample in a contact lensbased glucose sensor (Yang et al., 2006). Tear fluid also contains lipid, aqueous and mucin layers and has many functions including defending the ocular surface (Ohashi et al., 2006). Glucose measurements may also be taken from interstitial fluid (fluid between tissue cells) and a continuous measurement system has been developed by Abbott Laboratories Ltd. to provide early warning alarms of high or low glucose levels. The sensor can be worn for up to 5 days. The sensor tail is inserted $5 \mathrm{~mm}$ into the skin and transmits wirelessly to a receiver.

Of all these body fluids sweat is the most accessible within a garment structure. Sweat is a filtrate of blood plasma and contains many substances of interest such as sodium and chloride (Wilmore et al., 2008). Sodium concentration in sweat is typically $20-80 \mathrm{mM} / \mathrm{L}$ and chloride concentration is $20-60 \mathrm{mM} / \mathrm{L}$. Other substances exist in lower concentrations including potassium $(4-8 \mathrm{mM} / \mathrm{L})$, bicarbonate $(0-35 \mathrm{mM} / \mathrm{L})$, and calcium $(0-1 \mathrm{mM} / \mathrm{L})$ (Maughan, 2000). Sweat electrolyte concentration varies greatly between individuals, being affected by genetics, sweat rate, type of training, degree of hydration and the state of heat acclimatization.

Despite this, sweat rates and sweat composition can be used to diagnose certain health conditions. Sweating excessively, known as hyperhidrosis, or a reduced ability to sweat, known as anhidrosis, can be an indication of an underlying problem with the nervous system (Cheshire and Freeman, 2003). Correct diagnosis depends on determining the anatomical pattern of sweating. Cystic Fibrosis (CF) is a disease which affects the transfer of water and electrolyte to and from cells. Since its discovery in the 1950's, the gold standard test for CF is the sweat test, which measures the sodium and chloride concentration in the sweat of infants (Massie et al., 2000). Individuals with $\mathrm{CF}$ have higher than normal levels of sodium chloride in their sweat. Sweating is stimulated using pilocarpine iontophoresis and then collected and analysed. Wescor Inc. have developed a watch which links to a bench top system to measure sweat conductivity. Lynch et al used miniaturised solid-state ion-selective electrodes to analyse sweat samples for sodium, potassium and chloride simultaneously in real-time. The micro-sensor array was incorporated into a Wescor watch-like sweat sampler as a small, portable, analytical instrument (Lynch et al., 2000).

Sweat analysis has also been investigated for sports nutrition and performance, and it is known that maintaining fluid and electrolyte balance is essential for health (Maughan and Shirreffs, 2008). However the information available is somewhat limited due to the difficulties in collecting sweat for analysis. The most commonly employed approach to date involves the use of patches or pouches placed on the skin to gather a sample, followed by laboratory analysis using standard measurement techniques. This leads to a delay between sampling and analysis. There is also a risk of contamination of the sample and it may be difficult to ensure the integrity of collection. Sampling sweat from a single site on the body may not give an accurate representation of the total body electrolyte loss as there are regional variations in sweat composition and volume. Therefore multiple sampling sites are needed, and a recent study (Patterson et al., 2000) has identified four sampling regions (the chest, scapula, forearm and thigh) using surface area weighted equations to estimate mean whole body concentrations of sodium in sweat.

Electrolyte losses are a function of sweating rate and sweat composition. Both of these vary over time, and are also influenced by the exercise conditions and the physiology of the 
individual (Maughan, 2000). Therefore measurements need to be provided in real-time in order for the individual to manage their fluid and electrolyte needs. The need to place sensors at different locations on the body may be addressed by using integrated textile-based sensors which could form a body sensor network within a garment.

\subsection{Textile-based sweat analysis platform}

As part of the EU BIOTEX project a textilebased platform was developed to collect and analyze sweat in real-time on the body. A detailed description of this wearable sensing system has been previously reported (Coyle et al., 2010). From this work it was found that a priming time (10-20 minutes) was involved before sweat measurements became valid. This was partly due to the time needed for the subject to start sweating and then for sweat to enter the fluidic channel and reach the sensor (dead volume). While this depended on environmental conditions, it also meant that the sensor was unsuitable for subjects who were unable to partake in exercise for long periods of time. Clearly a smaller device with smaller dead volume would reduce the delay between commencing exercise and generating valid data and also make the device accessible for a wider range of user groups. Therefore we have developed a microchip version of the platform to measure changes in the $\mathrm{pH}$ of

(a) $200 \mu m$ wide channel

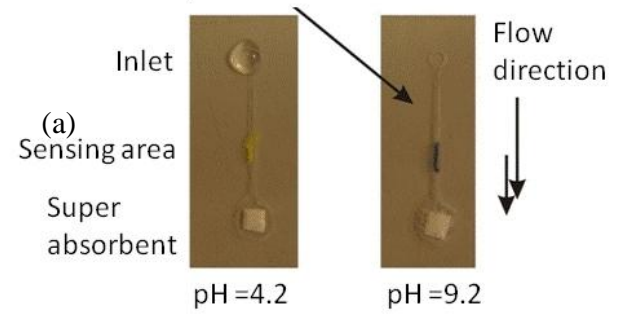

(b)

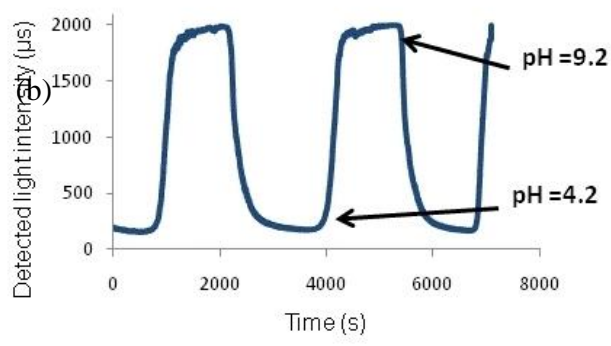

Figure 1 a) Microfluidic chip b) Calibration results of the microfluidic chip. sweat, shown in Figure 1(a). The device has been incorporated into a simple adhesive plaster format that can be easily attached to the skin thereby causing little or no discomfort during training. The micro-chip $(2 \times 3 \mathrm{~cm})$ was fabricated using extra thin poly(methylmethacrylate) $(50 \mu \mathrm{m})$ and pressure-sensitive adhesive $(80 \mu \mathrm{m})$ in three layers using a $\mathrm{CO}_{2}$ ablation laser. The inlet has a drop shape for efficient sweat collection when in contact with the skin. The sensing area was a small patch of $\mathrm{pH}$ sensitive fabric $(2 \mathrm{~mm} \times 2 \mathrm{~mm})$. The fabric was dyed with bromocresol purple dye which is sensitive to the $\mathrm{pH}$ range of sweat $(\mathrm{pH} 4-7)$. The sweat is drawn into the sensing area by a piece of superabsorbent non-woven (Absortex) placed at the end of the channel. The final device $(180 \mu \mathrm{m}$ thick) is flexible and can adapt easily to contours of the body.

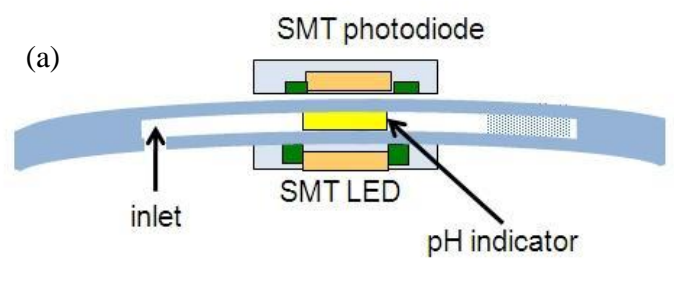

(b)

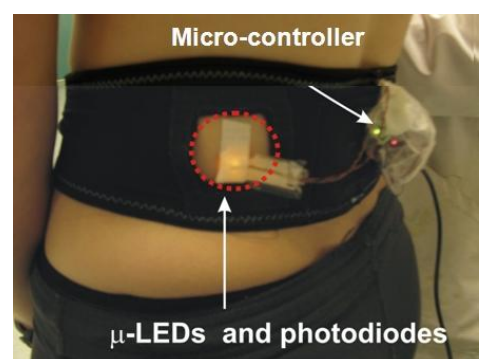

Figure 2 a) A photodiode and LED are placed above and below the $\mathrm{pH}$ sensitive region of the chip b) placing the sensor on the lower back during an exercise trial.

The color change of the $\mathrm{pH}$ sensitive fabric was detected by placing a surface mount (SMT) LED and phototodiode module on either side of the chip, aligned with the $\mathrm{pH}$ sensitive fabric (Benito-Lopez et al., 2009). Light is attenuated as it passes through the sensing region depending on the $\mathrm{pH}$ of the sample. The detected light signal was sampled by a 10bit analog to digital (A/D) channel of an Xbee transceiver (Digi International Inc.) at a rate of $2 \mathrm{~Hz}$. Data was wirelessly transferred to an Xbee base-station connected to a laptop using a USB serial link. The sensor response to $\mathrm{pH}$ solutions of $\mathrm{pH} 4.2$ and $\mathrm{pH} 9.2$ is shown in Figure 1(b). The LED and photodiode were coated with clear silicone to ensure effective sealing from moisture during on-body trials 
(Figure 2a). The device was attached to the lower back using surgical tape during experimental trials as shown in Figure 2(b). Figure 3 shows the response of the wearable $\mathrm{pH}$ sensor during an exercise trial. This exercise trial involved indoor cycling at self selected pace. The subject wore a physiological sensing vest, manufactured by QinetiQ, which monitored heart rate and respiration rate. Once the subject started to sweat sufficiently reference $\mathrm{pH}$ measurements were taken every 10 minutes using a skin $\mathrm{pH}$ meter. The measurements were taken by placing the electrode on a reference fabric patch, as performed in previous studies (Coyle et al., 2010). The subject was asked to stop cycling to reduce motion in order to take a stable reading. This resulted in a decrease in heart rate at these sampling times due to the reduced effort. The error between the reference measurement and the wearable sensor is less than $0.2 \mathrm{pH}$ units. The deviation in the wearable sensor after 32 minutes may have been due to motion artefacts and movement of the sensor away from the skin surface due to excessive sweat. Future studies will investigate motion effects by using a chip with a sensing region that does not change colour.

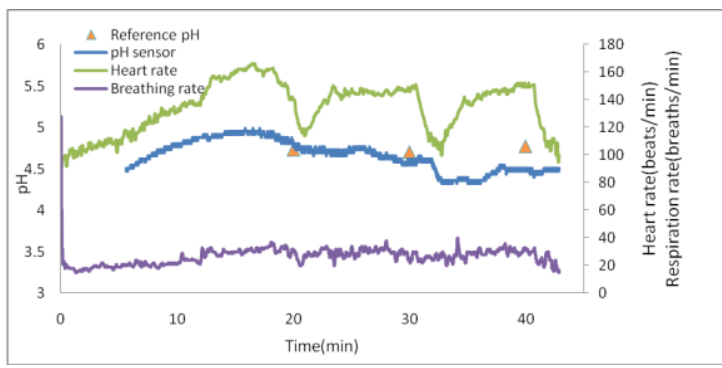

Figure 3 Performance of the wearable sweat sensor during an exercise trial. Reference $\mathrm{pH}$ measurements are taken along with heart rate and breathing rate data.

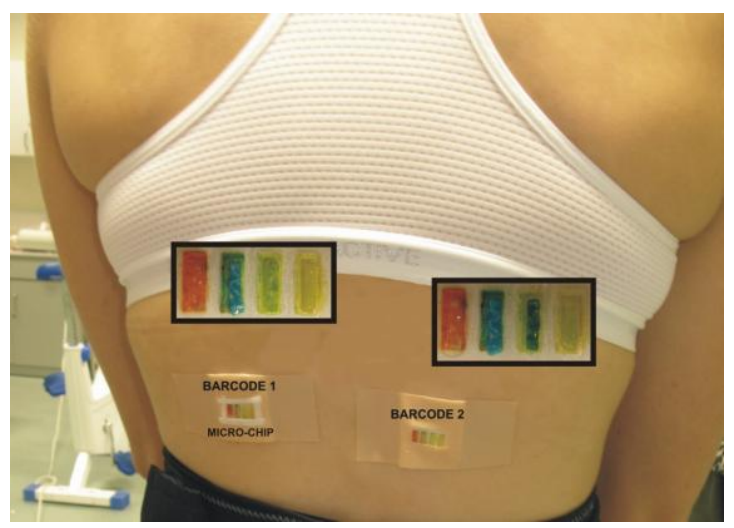

Figure 4 Barcode $\mathrm{pH}$ sensor

\subsection{Barcode $\mathrm{pH}$ sensors}

A variation of the wearable system for sweat analysis has been developed using colorimetric barcode sensors. The sensors use ionic liquids incorporated into polymer gels, to form ionogels that have $\mathrm{pH}$ dyes immobilized into the structure. The barcode system consisting of four independent reservoirs was fabricated in poly(methyl methacrylate) and pressuresensitive adhesive in five layers using a $\mathrm{CO}_{2}$ ablation laser. The outer layer contains four 1 $\mathrm{mm} \times 5 \mathrm{~mm}$ holes situated over the polymerised ionogels so that sweat entering at these points reacts with the ionogels. Four $\mathrm{pH}$ sensitive dyes that respond over different $\mathrm{pH}$ ranges were used - methyl red (MR), bromocresol green (BCG), bromocresol purple (BCP) and bromothymol blue (BTB). Figure 4 shows two barcodes placed on the middle back of an athlete during an exercise trial. The $\mathrm{pH}$ value of the sweat sample generates a unique colour barcode, which can be compared to a standard colour chart or analysed using camera/video analysis. BCG, BCP and BTB exhibited vivid colour changes in their sensitive $\mathrm{pH}$ range but the colour change of MR was not as visible. The response the barcode to $\mathrm{pH}$ levels from $\mathrm{pH} 1-14$ is shown in Figure 5. Analysis of the $\mathrm{pH}$ by eye gives an accuracy of $0.5 \mathrm{pH}$ units. For more sensitive quantitative measurements it is possible to integrate optical components into the device to continuously measure the $\mathrm{pH}$ of the sweat, as with the previous systems. There is also potential to monitor the colour change using video cameras which may already be in place for sports performance analysis.

For this study photos of the barcodes were taken at 10 minute intervals during an exercise trial. Figure 5 shows the colour change of each of the $\mathrm{pH}$ ionogel indicators at each sampling time. Colorimetric RGB analysis was performed after the trial using MATLAB. To establish reference measurements the average RGB value was calculated for each dye at $\mathrm{pH}$ levels from pH1-14. Then RGB values of each indicator were measured for each 10 minute time interval. The best fit for the $\mathrm{pH}$ value was assessed using the three dyes BCG, BCG and BCP using a least square error approach. Methyl red was not considered as this does not show significant colour change. The measured error was less than $0.9 \mathrm{pH}$ units. Figure 6 shows the barcode response and $\mathrm{pH}$ reference measurements taken during a $50 \mathrm{~min}$. trial. Lighting effects need to be investigated 
further to see their effects on accuracy. The alternative approach of integrating optical components would reduce the effects of ambient light.

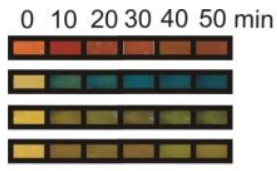

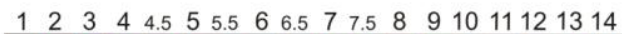
MR

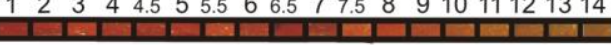

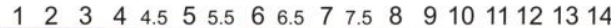

BCP

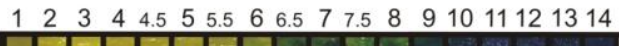

BTB

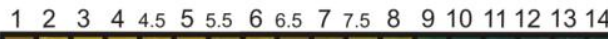

Figure 5 (above) Colour change of each indicator on the barcode during the 50min. exercise trial and (below) reference measurements for each indicator from $\mathrm{pH} 1-14$.

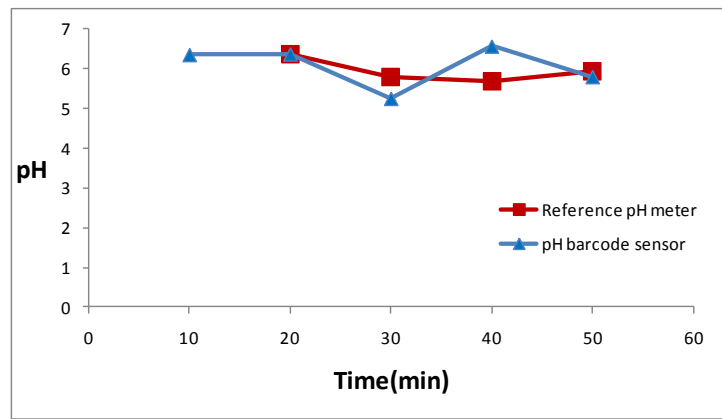

Figure 6 Sweat $\mathrm{pH}$ determination using the barcode in an athlete during a $50 \mathrm{~min}$. training period.

\section{Wearable chemical sensors "looking out" to identify hazards to the wearer}

PROETEX is an EU project which seeks to develop textile based sensors to improve the safety and efficiency of emergency workers. Part of this work involved the integration of carbon monoxide $(\mathrm{CO})$ and carbon dioxide $\left(\mathrm{CO}_{2}\right)$ sensors into garments for the safety of firefighters. High concentrations of these colourless, odourless gases pose a risk to human health as oxygen deprivation (arising from high levels of $\mathrm{CO}_{2}$ in air) can result in permanent brain damage, coma and even death, and $\mathrm{CO}$ is highly toxic, through displacement of oxygen from haemoglobin in the blood. It is therefore imperative for the health and operational effectiveness of firefighters that he/she is not over-exposed to these gases. Therefore a reliable method for monitoring $\mathrm{CO}$ and $\mathrm{CO}_{2}$ is needed to monitor an individual's degree of exposure. Integrating sensors into a protective smart garment could provide a warning when the concentrations of the gases is approaching a significant threshold, and warning the individual to go to a safer location. $\mathrm{A} \mathrm{CO}_{2}$ sensor has been integrated into the boot of a firefighter's uniform while a $\mathrm{CO}$ sensor has been integrated close to the jacket collar. The reason for placement was based on the densities of each gas $-\mathrm{CO}_{2}$ is denser than air and therefore tends to accumulate at lower layers, $\mathrm{CO}$ is of similar density to air therefore the sensor was placed close to the respiratory tract.

The $\mathrm{CO}$ and $\mathrm{CO}_{2}$ gas sensors have already an outer protective membrane built in to filter out small particles (dust, soot etc.). On the garment, the gas sensing devices are further protected by full encapsulation into a purposely designed pocket with a heat resistance, water-proof, air-permeable membrane; hence protecting them from potential damages from heat, water and airborne particles.

\section{$3.1 \mathrm{CO}_{2}$ sensor}

A potentiometric $\mathrm{CO}_{2}$ sensor (Alphasense Ltd.) was chosen for its low power demand and low cost (Radu et al., 2009). New generation miniaturized D-series $\mathrm{CO}_{2}$ sensors have diameters of $14.3 \mathrm{~mm}$ and heights of $13 \mathrm{~mm}$. The sensor contains a reference and working electrode placed in an electrolyte that provides a reference $\mathrm{CO}_{2}$ concentration. The measured potential is based on the difference in concentration between the reference electrode and the outside air. The sensor gives an accurate reading for concentrations ranging from $100 \mathrm{ppm}$ to more than $90 \% \mathrm{CO}_{2}$. This enables accurate detection of low concentration levels of $\mathrm{CO}_{2}$ (that may be hazardous over long exposure times) and high concentrations that pose immediate danger. The sensor has a lifetime of up to 12 months. A $3.7 \mathrm{~V}$ rechargeable battery was used to power the sensor, which allowed continuous operation for 5 hours. Wireless transmission was achieved using a $2.4 \mathrm{GHz}$ Zigbee module with an integrated ceramic antenna- Jennic JN5139-001-M00 802.15.4 RF Module. The signal is received by a base station (Jennic JN5139 RF Module sensor board) connected 
to a PC. The wireless sensing module shown in Figure 7 contains the integrated $\mathrm{CO}_{2}$ circuitry, $\mathrm{CO}_{2}$ sensor, battery and the Zigbee module. The module was placed into a customized protective casing before being placed inside of the boot pocket.

The module was first tested in a small airtight chamber of known volume. A range of $\mathrm{CO}_{2}$ concentrations from atmospheric to 42800 ppm were injected into the chamber using syringes. To ensure thorough mixing of gas within the chamber, a computer fan was placed inside of the chamber. The results of the calibration are shown in Figure 8. Following this test the module was placed into a waterproof, permeable membrane and tested in a larger chamber to simulate the real-life situation. Sensor sensitivity was reduced by $25 \%$ when placed in the boot pocket due to the formation of a concentration gradient arising from diffusion of gas across the membrane(Radu et al., 2009). Therefore the effects of laminate membranes must be considered when interating gas sensors into garments.

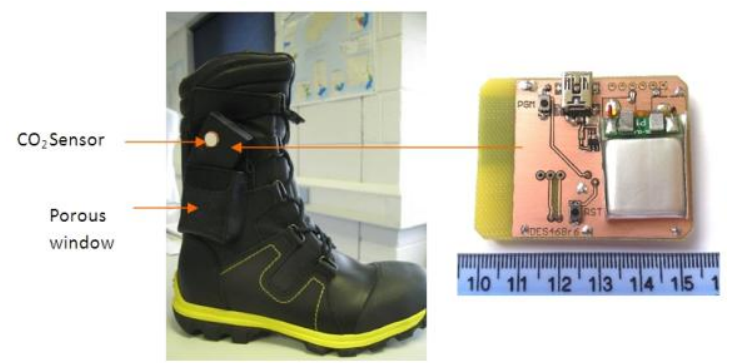

Figure 7 Firefighter's boot with built-in pocket used for enclosing the $\mathrm{CO}_{2}$ sensor and wireless sensing module

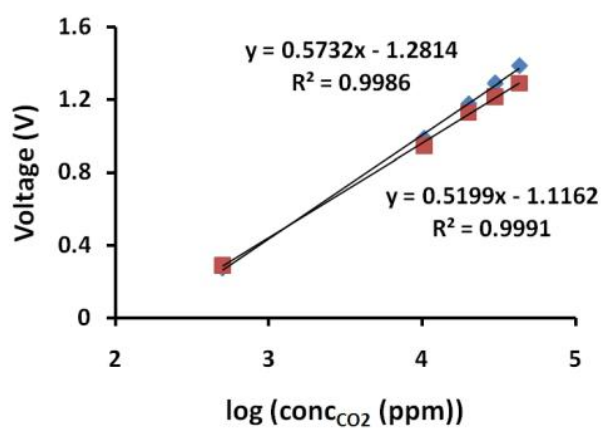

Figure 8 Calibration of $\mathrm{CO}_{2}$ sensor module in a small chamber. Calibration range: atmospheric to $42800 \mathrm{ppm}$
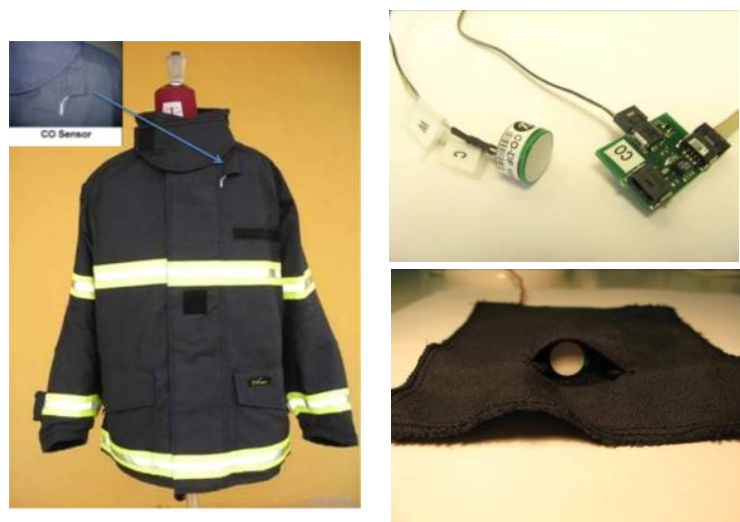

Figure $9-$ a) $\mathrm{CO}$ sensor integrated into the fire-fighter's jacket b) $\mathrm{CO}$ sensor connected to an on-body base station c) $\mathrm{CO}$ sensor inside the outer pocket of the jacket

\subsection{CO sensor}

An amperometric $\mathrm{CO}$ sensor (Alphasense CODF) was integrated into the jacket of a firefighter uniform, shown in Figure 9(a). The sensor has an excellent response time (less than $25 \mathrm{~s}$ from $0-400 \mathrm{ppm}$ ) and a wide operating range of $0-1000 \mathrm{ppm} \mathrm{CO}$. The sensor was connected to a sensing module which was connected to an on-body base station, shown in Figure 9(b). The base station receives signals from numerous on-body sensors and processes data via custom designed software. Alarms are activated if the concentration breaches a pre-defined threshold $\mathrm{CO}$ level of $50 \mathrm{ppm}$. Figure 10 shows a repeated multistep calibration of a typical $\mathrm{CO}$ sensor over the calibration range $0-400 \mathrm{ppm}$ at room temperature. These amperometric sensors have been shown to be very reproducible with error less than $5 \%(n=5)$. The $\mathrm{CO}$ sensor was located inside the outer pocket piece of the jacket, as shown in Figure 9(c) and placed inside a gas-tight chamber. $\mathrm{CO}$ gas was injected into the chamber using syringes and rapidly dispersed thoroughly by means of a computer fan placed inside of the chamber. The experiments were repeated using a second sensor and excellent correlation between the sensors' signals was obtained. The average response and recovery time of the sensors was below 1 minute, and the signals were very stable, with very low noise levels. 

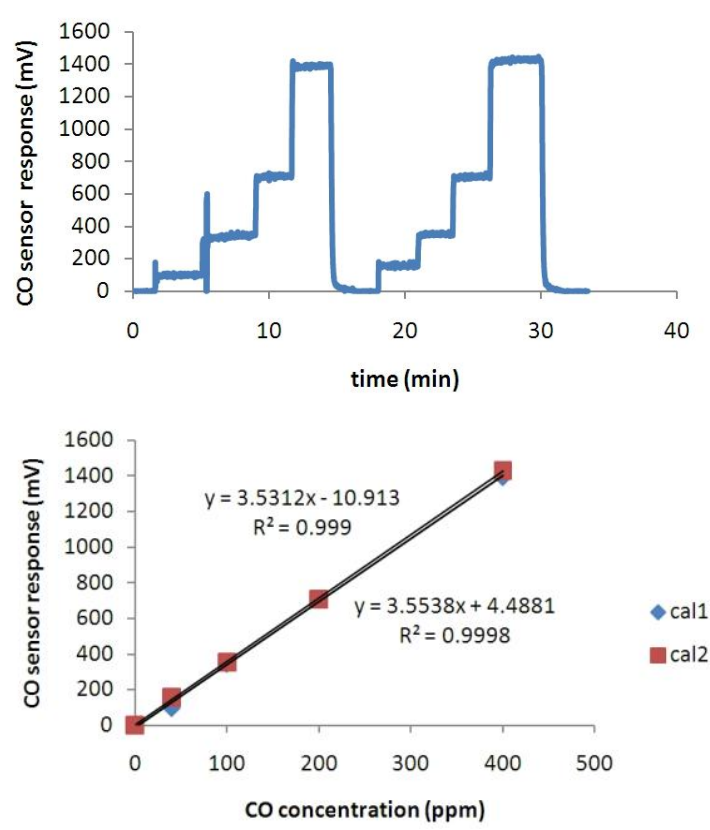

Figure $10 \mathrm{CO}$ sensor multistep calibration data for $\mathrm{CO}$ concentration range $0-400 \mathrm{ppm}$ (exposure to 0, 40, 100, 200 and $400 \mathrm{ppm}$ ) and room temperature. Top: raw experimental data, Bottom: linearized calibration plot

\section{Sensor integration}

\subsection{Electronic Textiles}

A recent survey on the wearable biosensor systems for health showed that wearability was a major issue because sensor, battery and onbody hardware size tend to be too bulky (Pantelopoulos and Bourbakis, 2008). We have reduced the $\mathrm{pH}$ sensor size from a channel length of $7 \mathrm{~mm}$ to $200 \mu \mathrm{m}$, and sensing region from $49 \mathrm{~mm}^{2}$ to $4 \mathrm{~mm}^{2}$. Reducing the sensor size helps to reduce power requirements and also makes the device less obtrusive for the wearer. In this case with a chemical sensor we also reduce the size of sample needed for the sensor to operate. However miniaturization brings added challenges to the manufacturing processes. Placement of the optical components needs to be very precise and movement artefacts can have a greater impact when the sensing region is reduced. The smaller inlet needs to have good contact with the skin surface in order to allow sweat to enter the channel. In initial studies the LED and photodiode were encased in a $2 \mathrm{~mm}$ layer of silicone but it was found during the experimental on-body trials that this added height caused the inlet to be lifted away from the skin. While this could be fixed by increasing the length of the channel this would also affect the response time of the system. Therefore we chose to protect the LED and photodiode by spraying with silicone spray, as typically used to insulate circuit boards to give a thinner insulated coating. The ideal solution would be to create a textile device using organic LEDs integrated into a flexible garment structure.

\subsection{Sensor developments}

Wearable sensors are similar to other sensors considering their development strategy. Essential sensor performfince characteristics such as detection range, accuracy, resolution and limit of detection (LOD) have to be satisfied for the specific applications. Generally, wearable sensors are required to be small, simple, light weight, flexible, easy to operate, low power alid have wireless transmission capability but they also need to produce reliable data in order to be useful in practice. This is because wearable sensors have to face very stringent challenges during operation which makes them fundamentally different from other deployable sensors. The main differences, for example, are that wearable sensors have to be safe and comfortable to wear, may need to be compatible with the life-style of the user and have a design to reduce motion artifacts and to be calibration free.

For wearable on-body chemical and biochemical sensing, good sensor design strategy is the key to success. It is important to design the sensor around the application, taking care to understand the requirement for sample collection and delivery, detection, data transmission and, most importantly, the selection of the most appropriate sensing technique.

One of the common disadvantages of chemical/biochemical sensors is the need to calibrate from time to time due to either small changes in the activity of the sensor itself and/or the contamination (fouling) of the sensor surface during operation. This has seriously limited the application of these sensors in continuous monitoring scenarios for long period of time (days-weeks). One way to circumvent this issue is to make the sensor low cost enough to be disposable (usually singleuse). The $\mathrm{pH}$ sensor is designed so that the sensing element is disposable. These are low cost microfluidic chips which could be mass produced. The optical sensing elements and wireless system are reusable. This approach does not completely eliminate the need for calibration; but the sensor can be pre- 
calibrated (e.g. factory calibrated) such that output can be corrected by using a pre-set calibration equation, typically based on the slope and baseline (zero point) of the response curve. After each exercise trial the microfluidic chip is calibrated using 2 solutions of $\mathrm{pH} 4$ and $\mathrm{pH} 7$.

To improve reproducibility between sensors the fabrication techniques need to be standarized. For example, the sensor size (area), thickness, and the amount of active reagent, have to be strictly controlled and reproducibly fabricated. In the case of the $\mathrm{pH}$ sensor a uniform distribution of dye must be ensured on the fabric with the same concentration between batches.

\section{Conclusions}

Chemical sensors involve a reaction with a particular substance which means that the sensing surface must have access to samples of this substance. In this paper, we have presented two approaches to integrate chemical sensors into wearable devices to monitor the health and well-being of the wearer. The first approach analyses fluids from the body through the sample and analysis of sweat from the body in real time. Microfluidic chip structures using colorimetric sensing elements have been developed for this purpose. Sweat $\mathrm{pH}$ has been analyzed in this study however the platform could be adapted to facilitate colorimetric reactions targeting other important analytes such as lactate and sodium (O'Neill et al., 1999).

The second application discussed in this article involves the use of chemical sensors to sense the environment around the wearer and thereby warn of any hazards that may be present. Carbon monoxide and carbon dioxide sensors were integrated into the uniform of a firefighter. This work was part of the EU ProeTEX project. Obtaining information about hazardous gases through continuous, real-time monitoring can clearly help in improving the safety and efficiency of workers in extreme environments.

Wearable chemosensors have an important role to play in citizen healthcare and ensuring personal safety. Low-power devices that require straightforward or no calibration by the user are needed. This may involve replacement of the sensing element, e.g. replacing the microchip but keeping the optical component configuration in the case of the sweat sensors. Therefore sensors must be easily fabricated and low-cost to manufacture. The sensors must also be robust and able to withstand extreme environments and of course comfortable and easy-use for the wearer.

\section{Acknowledgements}

This work is supported by Science Foundation Ireland under grant 07/CE/I1147 and the European Union under ProeTEX FP6-2004IST-4-06987.

\section{REFERENCES}

Benito-Lopez, F., Coyle, S., Byrne, R., Smeaton, A., O'Connor, N. \& Diamond, D. (2009) Pump Less Wearable Microfluidic Device for Real Time pH Sweat Monitoring. Eurosensors XXIII Conference. Lucerne, Switzerland, Procedia Chemistry.

Brady, S., Dunne, L., Lynch, A., Smyth, B. \& Diamond, D. (2006) Wearable Sensors? What is there to sense? Stud Health Technol Inform, vol. 117, no., pp.8088.

Cheshire, W. \& Freeman, R. (2003) Disorders of sweating. Seminars in Neurology, vol. 23, no. 4, pp.399-406.

Coyle, S., Lau, K., Moyna, N., Diamond, D., Di Francesco, F., Constanzo, D., Salvo, P., Trivella, M., De Rossi, D., Taccini, N., Paradiso, R., Porchet, J., Luprano, J., Ridolfi, A., Chuzel, C., Lanier, T., Revol-Cavalier, F., Schoumacker, S., Mourier, V., Convert, R., Chartier, I., De-Moncuit, H. \& Bini, C. (2010) BIOTEX - Biosensing textiles for personalised healthcare management. IEEE Transactions on Information Technology in BioMedicine, vol. 14, no. 2, pp.374-370.

Kaufman E, L. I. (2002) The diagnostic applications of saliva--a review. Critical Reviews in Oral Biology and Medicine, vol. 13, no. 2, pp.197-212.

Lee, J. M., Garon, E. \& Wong, D. T. (2009) Salivary diagnostics. Orthodontics and Craniofacial Research, vol. 12, no. 3, pp.206-211.

Lynch, A., Diamond, D. \& Leader, M. (2000)

Point-of-need diagnosis of cystic fibrosis using a potentiometric ionselective electrode array. The Analyst, vol. 125, no., pp.2264-2267. 
Massie, J., Gaskin, K., Van Asperen, P. \& Wilcken, B. (2000) Sweat testing following newborn screening for cystic fibrosis. Pediatric Pulmonology, vol. 29, no. 6, pp.452-456.

Maughan, R. (2000) Nutrition in sport, Malden, MA, Blackwell Science Ltd.

Maughan, R. J. \& Shirreffs, S. M. (2008) Development of individual hydration strategies for athletes. International Journal of Sport Nutrition and Exercise Metabolism, vol. 18, no. 5, pp.457-72.

O'Neill, S., Conway, S., Twellmeyer, J., O. Egan, Nolan, K. \& Diamond, D. (1999) Ion-selective optode membranes using 9-(4-diethylamino2-octadecanoatestyryl)-acridine acidochromic dye. Anal Chim Acta, vol. 398, no., pp.pp. 1-11.

Ohashi, Y., Dogru, M. \& Tsubota, K. (2006) Laboratory findings in tear fluid analysis. Clinica Chimica Acta, vol. 369, no., pp.17-28.

Pantelopoulos, A. \& Bourbakis, N. (2008) A Survey on Wearable Biosensor Systems for Health Monitoring. 30th Annual International Conference of the IEEE Engineering in Medicine and Biology. Vancouver.

Patterson, M., Galloway, S. \& Nimmo, M. A. (2000) Variations in regional sweat composition in normal human males. Experimental Physiology, vol. 85, no. 6, pp.869-876.

Peredy, T. R. \& Powers, R. D. (1997) Bedside Diagnostic Testing of Body Fluids. The American Journal of Emergency Medicine, vol. 15, no. 4, pp.400-407.

Radu, T., Fay, C., Lau, K., Waite, R. \& Diamond, D. (2009) Wearable sensing application- carbon dioxide monitoring for emergency personnel using wearable sensors. ICESE 2009 International Conference on Environmental Systems Engineering, 28-30 Oct 2009. Venice, Italy.

Wilmore, J. H., Costill, D. L. \& Kenney, W. L. (2008) Physiology of sport and exercise, Human Kinetics.

Yang, X., Pan, X., Blyth, J. \& Lowe, C. (2006) Towards the real-time monitoring of glucose in tear fluid: holographic glucose sensors with reduced interference from lactate and $\mathrm{pH}$.

Biosensors and Bioelectronics, vol. 23, no. 6, pp.899-905.

\section{Full mailing address of the corresponding author:}

(We will send the Journal to the mailing address if the paper is published. The address will be not shown in your paper)

Attn: Prof. Dermot Diamond

Mailing address:

Room L239B

CLARITY: Centre for Sensor Web Technologies

Dublin City University

Glasnevin

Dublin 9

Ireland 